\title{
The correlation between mood states and functional connectivity within the default mode network can differentiate Internet gaming disorder from healthy controls
}

Guangheng Dong 1,2*, Hui Li ${ }^{1}$, Lingxiao Wang 1, Marc N. Potenza ${ }^{3 *}$

${ }^{1}$ Department of Psychology, Zhejiang Normal University, Jinhua, P.R. China

${ }^{2}$ Institute of Psychological and Brain Sciences, Zhejiang Normal University, Jinhua, P.R. China

${ }^{3}$ Departments of Psychiatry and Neuroscience, Child Study Center, and the National Center on Addiction and Substance Abuse (CASAColumbia), Yale University School of Medicine, New Haven, CT, 06510, USA

${ }^{*}$ Corresponding authors:

Guangheng Dong, Ph.D. Professor

Department of Psychology, Zhejiang Normal University, 688 Yingbin Road, Jinhua, Zhejiang Province 321004, PR China. Tel.: +86 15867949909.

Email: dongguangheng@zjnu.edu.cn

Marc N. Potenza, Ph.D., M.D., Professor

Department of Psychiatry and Neurobiology, Child Study Center, CASAColumbia, Yale University School of Medicine, New Haven, CT, 06510, USA

Email: marc.potenza@yale.edu 


\section{Abstract}

The default-mode network (DMN) has been suggested to support a baseline state of brain activity. However, whether connectivity within the DMN is associated with mood states remains incompletely understood. The current study examined the correlation between mood state and the functional connectivity (FC) among DMN regions, and examined if the FC can differentiate Internet gaming disorder (IGD) from healthy controls (HC). Resting state data were collected within 108 college students (IGD,41; HC,67). Negative correlations were observed between measures of: (1) Depression and FCs among ventral DMN regions; (2) Anger and FCs among dorsal DMN regions; and, (3) Anger and Depression and FCs of both the ventral and dorsal DMN. The results suggest that negative mood states of Depression and Anger might reflect poorer, or might impair, FCs among DMN regions. In addition, the FC among DMNs could be useful indexes in differencing IGD from HC. Future studies should examine the extent to which the findings may extend to clinical populations and whether increased connectivity of DMN regions may represent a mechanism for reducing negative mood states.

Key Words: default mode network; mood state; resting states fMRI; functional connectivity 


\section{Introduction}

The default-mode network (DMN) is an anatomically defined brain system that preferentially activates when individuals are not focused on the external environment (Buckner et al, 2008; Raichle et al, 2001). The core areas of the DMN include regions of the posterior cingulate cortex (PCC), precuneus, and medial prefrontal cortex (Buckner et al, 2008; Gusnard et al, 2001; Raichle et al, 2001). Recent studies suggest that the DMN is comprised of two distinct systems: the dorsal DMN, which includes dorsal and anterior regions; and the ventral DMN, which involves posterior and mesial temporal regions. These two components were found to dissociate functionally, and thus these two systems may represent distinct subsystems (Andrews-Hanna et al, 2010b; Doucet et al, 2011; Migliaccio et al, 2016; Shirer et al, 2012). Although the DMN typically refers to functionally connected brain regions measured in the resting state, it may also serve important cognitive functions (Andrews-Hanna et al, 2010a; Sheline et al., 2010a; Dong et al, 2015a; Dong et al, 2015b; Greicius et al, 2003; Kinnison et al, 2012). In addition, the failure to successfully involve different brain regions in the DMN has been associated with multiple psychological and psychiatric disorders (Iordan and Dolcos, 2015; Sheline et al, 2010b).

Regions within the DMN interact when people perform tasks and are at rest. The brain connectivity among DMN regions permits exploration of interactions between different brain regions and can be used as indexes to mark cognitive 
functions (Dong et al, 2015b; Friston, 2011; Kahan et al, 2014; Riedl et al, 2016). Functional connectivity (FC) of the DMN may reflect experience-dependent plasticity that may form a foundation for subsequent activity of forthcoming stimuli (Iordan et al, 2015; Kinnison et al, 2012; Li et al, 2014).

Moods are subjective mental states that may last for relatively long times. Compared to emotions, moods may be less intense, less specific, and less likely to be triggered by a particular event (Jackson and Arlegui-Prieto, 2016). Moods are typically described as having either a good/positive or bad/negative valence, and moods, especially negative ones, have important implications for human mental and psychological wellbeing (Dhuffar et al, 2015; Foland-Ross et al, 2012; Jackson et al, 2016; Prossin et al, 2016). It is possible that the FC of the DMN may relate to mood states. The DMN is active when a person is not focused on the outside world and the brain is at wakeful rest; mood reflects emotional states not stimulated by events. Thus, both may involve mental states without outside disturbances. Second, both the DMN and mood states are relevant to psychiatric disorders such as depression (Ho et al, 2015) and addiction (Liang et al, 2015). Studies have also linked individual differences in depressive mood changes and serotonin-depletion-induced brain changes in the DMN (Kunisato et al, 2011). Thus, mood states and DMN FCs may be linked. Third, both the DMN and mood states may reflect "mental baselines" over a relatively long period of time, with DMN FCs reflecting the default neural activities of the brain and mood states 
reflecting baseline mental states. Thus, exploring the relationships between DMN FCs and mood warrants consideration.

Internet gaming disorder (IGD) has been conceptualized as a behavioral addiction (Dong et al., 2011; Dong and Potenza, 2014, 2016; Dowling, 2014; Petry and O'Brien, 2013; Petry et al, 2015; Rehbein et al, 2015; Dong et al.,2017a), although no chemical intake is needed for it. IGD has also demonstrated neurobiological similarities to substance-use and gambling disorders (Liu et al, 2016; Park et al, 2016; Yuan et al, 2016). Studies have showed that IGD are usually parallel with negative features, such as depression, ADHD, withdraw, and so on (Kuss DJ, 2016; Dong et al., 2017b). Thus, the mood states and its correlation with functional connectivity might be in useful feature that can be observed in IGD subjects.

Based on the above rationale, the current study first sought to explore correlations between mood states and FCs in the DMN. As poorer interactions between regions of the DMN have been associated with negative emotions and psychiatric conditions (Ho et al, 2015; Liang et al, 2015; Patriat et al, 2016), we hypothesized that negative mood states would correlate inversely with DMN FCs. Second, this study is try to examine if the index is useful in differentiating IGD from HC. 


\section{Methods}

\section{Participants}

The experiment conformed to The Code of Ethics of the World Medical Association (Declaration of Helsinki). The Human Investigations Committee of Zhejiang Normal University approved this research. Data were collected from 108 (IGD,41; HC,67) university male students $20.2 \pm 1.7$ years) who were recruited through advertisements. All participants provided written informed consent and underwent structured psychiatric interviews (using the MINI) (Lecrubier et al, 1997) performed by an experienced psychiatrist. All participants were free of psychiatric disorders (including major depression, anxiety disorders, schizophrenia, and substance dependence disorders) as assessed by the MINI. IGD was determined based on scores of 50 or more on modified Young's online Internet addiction test (IAT) (focusing on Internet gaming) (Young, 2009) (Table 1) and, at the same time, meeting the proposed 9-item IGD diagnosis per DSM-5 criteria ( $\geq 5$ of 9) (Petry et al, 2014).

\section{Profile of Mood States (PoMS) Scale}

Mood states were measured using the PoMS scale (McNair et al, 1992). The scale is a series of 65-adjectives measuring six mood states: tension-anxiety (Tension), depression-dejection (Depression), anger-hostility (Anger), fatigue-inertia (Fatigue), vigor-activity (Vigor), and confusion-bewilderment (Confusion) on a five-point Likert scale from 0 (not at all) to 4 (extreme). Greater values for PoMS 
variables represent greater perceived mood states. The Total Mood Disturbance was calculated using [(Tension + Depression + Anger + Fatigue + Confusion $)-$ Vigor].

\section{Imaging data collection}

Scanning was performed and MRI data were acquired using a Siemens Trio 3T scanner (Siemens, Erlangen, Germany) in the MRI center of the East-China Normal University. Structural images were collected using a T1-weighted three-dimensional spoiled gradient-recalled sequence covering the whole brain (176 slices, repetition time $=1700 \mathrm{~ms}$, echo time $\mathrm{TE}=3.93 \mathrm{~ms}$, slice thickness $=1.0$ $\mathrm{mm}$, skip=0 mm, flip angle $=15$, inversion time $=1100 \mathrm{~ms}$, field of view $=240 * 240 \mathrm{~mm}$, in-plane resolution $=256 * 256$ ). Functional MRI was performed on a 3T scanner (Siemens Trio) with a gradient-echo EPI T2* weighted sensitive pulse sequence in 33 slices (interleaved sequence, $3 \mathrm{~mm}$ thickness, TR=2000ms, TE=30ms, flip angle $90^{\circ}$, field of view $220 \times 220 \mathrm{~mm}^{2}$, matrix 64x64). Resting state involved performance of no specific cognitive task during fMRI. Participants were instructed to keep still, close their eyes, remain awake and not to think of anything systematically (Zang et al, 2004). The resting-state functional images were acquired by using an EPI (echo-planar imaging) sequence. The resting-state fMRI scan lasted for 7 minutes (210 volumes).

\section{Data pre-processing}


The resting-state data analysis was performed using REST and DPARSF (http://restfmri.org) (Yan and Zang, 2010). Preprocessing consisted of removal of the first 10 time points (due to signal equilibrium and to allow the participants to adapt to the scanning noise), physiological correction, slice timing, volume registration and head-motion correction. We further calculated the group-difference in head motion parameters (Yan et al., 2013). No significant group-difference was found in six head motion direction $\left(p_{1}=0.64 \mathrm{t}_{1}=0.04\right.$, $p_{2}=0.36 \mathrm{t}_{2}=-0.62, p_{3}=0.65 \mathrm{t}_{3}=-1.28, p_{4}=0.56 \mathrm{t}_{4}=0.88, p_{5}=0.79 \mathrm{t}_{5}=0.98, p_{6}=0.92$ $\left.t_{6}=0.17\right)$. Possible contamination from several nuisance signals including signal relating to white matter, cerebral spinal fluid, global signal, and six motion vectors were regressed out (Tao et al., 2013; Wee et al., 2013; Ko et al., 2014; Wang et al., 2016). The time series of images of each subject were motion-corrected using a least-squares approach and a six-parameter (rigid-body) linear transformation (Friston et al, 1995). The individual structural image was co-registered to the mean functional image after motion correction using a linear transformation. The motion-corrected functional volumes were spatially normalized to the MNI (Montreal Neurological Institute) space and re-sampled to 3-mm isotropic voxels using the normalization parameters estimated during unified segmentation. Further preprocessing included band-pass filtering between 0.01 and $0.08 \mathrm{~Hz}$.

\section{Selection of Regions of Interest (ROIs)}


As the DMN is an anatomically defined brain system (Buckner et al, 2008; Raichle et al, 2001), regions of interest (ROIs) were chosen based on findings rather than by deriving seed regions from tasks in order to avoid bias and to increase the generalizability of findings (Duncan, 2013; Mansouri et al, 2009). In the current study, the DMN templates were downloaded from Stanford's Functional Imaging in Neuropsychiatric Disorders lab (Shirer et al, 2012) (see http://findlab.stanford.edu/functional ROIs.html). The DMN networks include two distinct networks: One is composed of dorsal and anterior regions, referred to as the dorsal DMN; while the other involves the more posterior regions, referred to as the ventral DMN (Andrews-Hanna et al, 2010b; Doucet et al, 2011; Migliaccio et al, 2016; Shirer et al, 2012). According to the definition and the templates, we selected 4 main brain regions as ROIs for the ventral DMN: the PCC, part of the precuneus, the medial frontal gyrus (MFG) and the medial temporal gyrus (Figure 1 a); At the same time, we selected 4 main brain regions as the ROIs for the dorsal DMN: the PCC, the MFG (different from the region in ventral DMN), the medial cingulate gyrus and the right thalamus (Figure $1 \mathrm{~b}$ ).

\section{Insert Figure 1 about here}

\section{Functional connectivity calculation}

For each ROI, a representative BOLD time course was obtained by averaging the signal of all the voxels within the ROI. Correlations between these two time 
courses were calculated as a functional connection index. Mean values were calculated as indexes when there are several FC values. Then, the correlation between the seven scores ( 6 subscales scores and the total score) of PoMS and the indexes of FC in the ventral, dorsal and combined DMN were calculated. The Bonferroni corrections were performed based on different mood states, separately.

\section{Results}

\section{Correlation between mood states and FC of the ventral DMN}

A negative correlation between FC of the ventral DMN and the total PoMS score approached but did not reach statistical significance $(r=-0.188, p=0.052)$. A negative correlation was found between the PoMS Depression scores and FC among ventral DMN brain regions $(r=-0.243, p=0.011)$. The relationship between FC of the ventral DMN did not reach significance for other components of the PoMS, with the relationship with Anger being the next most robust statistically $(r=-0.177, p=0.068)$ (See Figure 2).

\section{Insert Figure 2 about here}

\section{Correlations between mood states and FC within the dorsal DMN}

In the dorsal DMN, a negative correlation was found between Anger scores and FC among dorsal DMN brain regions ( $r=-0.208, p=0.031)$. No other significant 
correlations were found between FC within the dorsal DMN and other PoMS measures (See Figure 3).

\author{
Insert Figure 3 about here
}

\title{
Correlations between mood states and FC within the combined (ventral and
} dorsal) DMN

Negative correlations were found between FC of the ventral and dorsal DMN and Depression $(r=-0.204, p=0.034)$ and Anger $(r=-0.225, p=0.020)$ scores. A negative correlation between FC of the ventral and dorsal DMN and the PoMS total score did not reach statistical significance $(r=-0.172, p=0.075)$ (See Figure 4).

\section{Insert Figure 4 about here}

\section{The effect of these indexes in differentiating IGD from HC}

In behavioral, IGD subjects show higher scores in Tension, Depression, Anger, Fatigue, Confusion, except the Vigor factor (Figure 5a).

When we comparing IGD to HC in these indexes (FC among ventral and dorsal DMN), we found that IGD subjects show decreased FC indexes than HC in ventral, dorsal and ventral+dorsal aspects (Figure 5b).

\section{Insert Figure 5 about here}

\section{Discussion}

To our best knowledge, this is the first study exploring the correlation between 
mood states and FCs within the DMN, and explore if they can separate IGD from HC. As hypothesized, individual differences in some mood states were associated with FCs in the DMN. Specifically, negative correlations were found between Depression and Anger scores and FCs within the overall DMN, with the Depression scores linked particularly to the FC of the ventral DMN and Anger scores linked to the FC of the dorsal DMN.

Previous studies have suggested that the DMN is related to social and emotional processing. From a structural perspective, medial frontal areas, the thalamus, and the PCC have been linked to both generation and modulation of emotion (Kober et al, 2008; Lindquist et al, 2015; Wager et al, 2008) and are described as key regions of the DMN (Buckner et al, 2008; Gusnard et al, 2001; Raichle et al, 2001). Some of these regions have also been reported as being sensitive to emotional arousal and valence (Kober et al, 2008; Lindquist et al, 2015; Wager et al, 2008). Second, the DMN has been suggested to support a baseline state of brain activity and be involved in rudimentary functions (Raichle et al, 2001; Shirer et al, 2012). Distinct affective states and subclinical mood symptoms appear to have dissociable neural signatures, and there may exist a symbiotic relationship between cognitive processes and emotional states (Petrican et al, 2015). On the other hand, depressed people have altered DMN patterns during implicit emotional processing, suggesting a link between DMN FC and impaired abilities to regulate emotions in clinical populations (Shi et al, 2015). Thus, in the 
current study, the negative correlation between negative mood states and FCs among DMN regions resonates with prior findings. However, the extent to which negative mood states may disturb the baseline state of brain activity and lower interactions among DMN regions versus poorer FCs within the DMN leading to negative mood states warrants further examination.

In addition to emotion-related states, the DMN has also been linked to biological measures, personality traits, psychological features and psychiatric disorders. In current study, the FC among DMNs could separate IGD from HC effectively. A recent study proposed that resting state fMRI could be viewed as a potential biomarker of quantitative state changes in glucose metabolism (Thompson et al, 2016). FC among DMN regions has been associated with extraversion scores and other personality traits (Lei et al, 2013; Wei et al, 2014) and psychiatric disorders (Liang et al, 2015). Poorer connectivity of the DMN has been associated with adolescent depression (Ho et al, 2015), posttraumatic stress disorder (Patriat et al, 2016), and cocaine addiction (Liang et al, 2015). As existed in IGD, these disorders have also been characterized to varying extents by difficulties in emotional regulation. As mood may affect cognitive functioning, studies should also examine the broader impact of decreased FC within the DMN in healthy and psychiatric populations (Dhuffar et al, 2015; Foland-Ross et al, 2012; Jackson et al, 2016; Prossin et al, 2016). As mindfulness meditation training may increase DMN FC (Creswell et al, 2016), future studies may also 
examine the extent to which such interventions may be helpful for psychiatric conditions and operate through changes in FC of the DMN.

Limitations of the current study include the extent to which the findings extend to other populations (e.g., differing in age and cultural/racial/ethnic backgrounds) warrants direct investigation. Second, the global signal is a controversial resting state fMRI pre-processing option (Murphy et al.,2016). The use of global signal regression might smear connectivity differences between groups in other studies (Chai et al., 2012; Greicius et al., 2007; Veer et al., 2010). Whether global signal regression has complex effects on correlation still remains unclear in resting state research. This fact should be taken into account when interpreting results. Third, only male participants were included in the study, resulting in some limitations with our conclusion. All of these need to be investigated further in the future. More future studies are expected to examine the results of this study.

In summary, the current study provides evidence for an association between individual differences in mood states and FC within the DMN. Specifically, the higher the PoMS score in Depression and Anger, the lower the FCs among DMN regions. The current results deepen our knowledge about the DMN and its relationships with mental states. In addition, the FC among DMN could be a useful index in differencing IGD from HC. Future studies should explore the 
directional relationship between DMN FCs and mood states, whether the findings extend to specific clinical populations and whether specific interventions might operate to improve mood states via changes in FCs among DMN regions. 


\section{Contributors}

Guangheng Dong designed this research and wrote the first draft of the manuscript. Hui Li and Lingxiao Wang contributed to data collection, data analysis, and figure preparation. Marc Potenza contributed in interpreting findings, editing, and revisions. All authors contributed to and have approved the final manuscript.

\section{Competing Interests}

The authors declare that no competing interests exist.

\section{Funding and Disclosure}

Dr. Dong was supported by the National Science foundation of China (31371023). Dr. Potenza's involvement was supported by NIDA (R01 DA035058), CASAColumbia and the National Center for Responsible Gaming. The funding agencies did not contribute to the experimental design or conclusions, and the views presented in the manuscript are those of the authors and may not reflect those of the funding agencies. The authors report no conflicts of interest with respect to the content of this manuscript. Dr. Potenza has consulted for and advised Ironwood, Lundbeck, INSYS, Shire, RiverMend Health and Lakelight Therapeutics/Opiant; has received research support from Mohegan Sun Casino, the National Center for Responsible Gaming, and Pfizer; has participated in surveys, mailings or telephone consultations related to drug addiction, impulse-control disorders or other health topics; has consulted for gambling and legal entities on issues related to impulse-control and addictive disorders; provides clinical care in the Connecticut Department of Mental Health and Addiction Services Problem Gambling Services Program; has performed grant reviews for the 
National Institutes of Health and other agencies; has edited journals or journal sections; has given academic lectures in grand rounds, CME events and other clinical or scientific venues; and has generated books or book chapters for publishers of mental health texts. All other authors declare no financial interests. 


\section{References}

Andrews-Hanna JR, Reidler JS, Sepulcre J, Poulin R, Buckner RL (2010a).

Functional-Anatomic Fractionation of the Brain's Default Network. Neuron 65(4): 550-562.

Andrews-Hanna JR, Reidler JS, Sepulcre J, Poulin R, Buckner RL (2010b).

Functional-anatomic fractionation of the brain's default network. Neuron 65(4): $550-562$.

Buckner RL, Andrews-Hanna JR, Schacter DL (2008). The brain's default network: anatomy, function, and relevance to disease. Annals of the New York Academy of Sciences 1124: 1-38.

Creswell JD, Taren AA, Lindsay EK, Greco CM, Gianaros PJ, Fairgrieve A, et al (2016). Alterations in Resting-State Functional Connectivity Link Mindfulness Meditation With Reduced Interleukin-6: A Randomized Controlled Trial. Biological psychiatry.

Chai, X. J., Castañón, A. N., Ongür, D., \& Whitfieldgabrieli, S. (2012). Anticorrelations in resting state networks without global signal regression. Neuroimage, 59(2), 1420-8.

Dhuffar MK, Pontes HM, Griffiths MD (2015). The role of negative mood states and consequences of hypersexual behaviours in predicting hypersexuality among university students. J Behav Addict 4(3): 181-188.

Dong, G., Huang, J., \& Du, X. (2011). Enhanced reward sensitivity and decreased loss sensitivity in internet addicts: an fmri study during a guessing task. Journal of Psychiatric Research, 45(11), 1525-9.

Dong G., Li H., Wang L., Potenza M (2017a). Cognitive Control and Reward/Loss Processing in Internet gaming disorder: Results from the comparison with Recreational Internet Game-Users. European Psychiatry, Accepted. 
Dong G, Lin X, Potenza MN (2015a). Decreased functional connectivity in an executive control network is related to impaired executive function in Internet gaming disorder. Progress in neuro-psychopharmacology \& biological psychiatry 57: 76-85.

Dong G., Wang L, Du X, Potenza MN (2017b).Gaming increases craving to gaming-related stimuli in individuals with Internet gaming disorder. Biological Psychiatry: CNNI, accepted

Dong G, Potenza MN (2014). A cognitive-behavioral model of Internet gaming disorder: theoretical underpinnings and clinical implications. Journal of psychiatric research 58: 7-11.

Dong G, Potenza MN (2016). Risk-taking and risky decision-making in Internet gaming disorder: Implications regarding online gaming in the setting of negative consequences. Journal of psychiatric research 73: 1-8.

Dong GH, Lin X, Hu YB, Xie CM, Du XX (2015b). Imbalanced functional link between executive control network and reward network explain the online-game seeking behaviors in Internet gaming disorder. Scientific reports $\mathbf{5}$.

Doucet G, Naveau M, Petit L, Delcroix N, Zago L, Crivello F, et al (2011). Brain activity at rest: a multiscale hierarchical functional organization. Journal of neurophysiology 105(6): 2753-2763.

Dowling NA (2014). Issues raised by the DSM-5 internet gaming disorder classification and proposed diagnostic criteria. Addiction 109(9): 1408-1409.

Duncan J (2013). The Structure of Cognition: Attentional Episodes in Mind and Brain. Neuron 80(1): 35-50.

Foland-Ross LC, Brooks JO, 3rd, Mintz J, Bartzokis G, Townsend J, Thompson PM, 
et al (2012). Mood-state effects on amygdala volume in bipolar disorder. Journal of affective disorders 139(3): 298-301.

Friston KJ (2011). Functional and effective connectivity: a review. Brain connectivity 1(1): 13-36.

Friston KJ, Frith CD, Frackowiak RS, Turner R (1995). Characterizing dynamic brain responses with fMRI: a multivariate approach. NeuroImage 2(2): 166-172.

Greicius, M. D., Flores, B. H., Menon, V., Glover, G. H., Solvason, H. B., \& Kenna, H., et al. (2007). Resting-state functional connectivity in major depression: abnormally increased contributions from subgenual cingulate cortex and thalamus. Biological Psychiatry, 62(5), 429-437.

Greicius MD, Krasnow B, Reiss AL, Menon V (2003). Functional connectivity in the resting brain: a network analysis of the default mode hypothesis. Proceedings of the National Academy of Sciences of the United States of America 100(1): 253-258.

Gusnard DA, Akbudak E, Shulman GL, Raichle ME (2001). Medial prefrontal cortex and self-referential mental activity: relation to a default mode of brain function. Proceedings of the National Academy of Sciences of the United States of America 98(7): 4259-4264.

Hong, S. B., Zalesky, A., Cocchi, L., Fornito, A., Choi, E. J., Kim, H. H., Suh, J. E., Kim, C. D., Kim, J. W., Yi, S. H,. \& Yi, S. H. (2013). Decreased functional brain connectivity in adolescents with internet addiction. PloS one, 8(2), e57831.

Ho TC, Connolly CG, Henje Blom E, LeWinn KZ, Strigo IA, Paulus MP, et al (2015). Emotion-Dependent Functional Connectivity of the Default Mode Network in Adolescent Depression. Biological psychiatry 78(9): 635-646.

Iordan AD, Dolcos F (2015). Brain Activity and Network Interactions Linked to 
Valence-Related Differences in the Impact of Emotional Distraction. Cerebral cortex.

Jackson MC, Arlegui-Prieto M (2016). Variation in normal mood state influences sensitivity to dynamic changes in emotional expression. Emotion 16(2): 145-149.

Kahan J, Urner M, Moran R, Flandin G, Marreiros A, Mancini L, et al (2014). Resting state functional MRI in Parkinson's disease: the impact of deep brain stimulation on 'effective' connectivity. Brain : a journal of neurology 137 : 1130-1144.

Kinnison J, Padmala S, Choi JM, Pessoa L (2012). Network analysis reveals increased integration during emotional and motivational processing. The Journal of neuroscience : the official journal of the Society for Neuroscience 32(24): 8361-8372.

Kober H, Barrett LF, Joseph J, Bliss-Moreau E, Lindquist K, Wager TD (2008). Functional grouping and cortical-subcortical interactions in emotion: A meta-analysis of neuroimaging studies. NeuroImage 42(2): 998-1031.

Kunisato Y, Okamoto Y, Okada G, Aoyama S, Demoto Y, Munakata A, et al (2011). Modulation of default-mode network activity by acute tryptophan depletion is associated with mood change: A resting state functional magnetic resonance imaging study. Neurosci Res 69(2): 129-134.

Kuss DJ L-FO (2016). Internet addiction and problematic Internet use: A systematic review of clinical research. World J Psychiatr 6(1): 143-176.

Lecrubier Y, Sheehan DV, Weiller E, Amorim P, Bonora I, Harnett Sheehan K, et al (1997). The Mini International Neuropsychiatric Interview (MINI). A short diagnostic structured interview: reliability and validity according to the CIDI. European Psychiatry 12(5): 224-231. 
Lei X, Zhao ZY, Chen H (2013). Extraversion is encoded by scale-free dynamics of default mode network. NeuroImage 74: 52-57.

Li W, Mai X, Liu C (2014). The default mode network and social understanding of others: what do brain connectivity studies tell us. Frontiers in human neuroscience 8: 74.

Liang X, He Y, Salmeron BJ, Gu H, Stein EA, Yang YH (2015). Interactions between the Salience and Default-Mode Networks Are Disrupted in Cocaine Addiction. Journal of Neuroscience 35(21): 8081-8090.

Lindquist KA, Satpute AB, Wager TD, Weber J, Barrett LF (2015). The Brain Basis of Positive and Negative Affect: Evidence from a Meta-Analysis of the Human Neuroimaging Literature. Cerebral cortex.

Liu L, Yip SW, Zhang JT, Wang LJ, Shen ZJ, Liu B, et al (2016). Activation of the ventral and dorsal striatum during cue reactivity in Internet gaming disorder. Addiction biology.

Mansouri FA, Tanaka K, Buckley MJ (2009). Conflict-induced behavioural adjustment: a clue to the executive functions of the prefrontal cortex. Nature Reviews Neuroscience 10(2): 141-152.

McNair DM, Lorr M, Droppleman LF (1992). Manual for the Profile of Mood States. . Educational and Industrial Service, San Diego.

Migliaccio R, Gallea C, Kas A, Perlbarg V, Samri D, Trotta L, et al (2016). Functional Connectivity of Ventral and Dorsal Visual Streams in Posterior Cortical Atrophy. Journal of Alzheimer's disease : JAD.

Murphy, K., \& Fox, M. D. (2016). Towards a consensus regarding global signal regression for resting state functional connectivity mri. Neuroimage. 
Park M, Choi JS, Park SM, Lee JY, Jung HY, Sohn BK, et al (2016). Dysfunctional information processing during an auditory event-related potential task in individuals with Internet gaming disorder. Translational psychiatry 6: e721.

Patriat R, Birn RM, Keding TJ, Herringa RJ (2016). Default-Mode Network Abnormalities in Pediatric Posttraumatic Stress Disorder. Journal of the American Academy of Child and Adolescent Psychiatry 55(4): 319-327.

Petrican R, Saverino C, Shayna Rosenbaum R, Grady C (2015). Inter-individual differences in the experience of negative emotion predict variations in functional brain architecture. NeuroImage 123: 80-88.

Petry NM, O'Brien CP (2013). Internet gaming disorder and the DSM-5. Addiction 108(7): 1186-1187.

Petry NM, Rehbein F, Gentile DA, Lemmens JS, Rumpf HJ, Mossle T, et al (2014). An international consensus for assessing internet gaming disorder using the new DSM-5 approach. Addiction 109(9): 1399-1406.

Petry NM, Rehbein F, Ko CH, O'Brien CP (2015). Internet Gaming Disorder in the DSM-5. Current psychiatry reports 17(9): 72.

Power, J. D., Barnes, K. A., Snyder, A. Z., Schlaggar, B. L., \& Petersen, S. E. (2012). Spurious but systematic correlations in functional connectivity mri networks arise from subject motion. Neuroimage, 59(3), 2142-2154.

Prossin AR, Koch AE, Campbell PL, Barichello T, Zalcman SS, Zubieta JK (2016). Acute experimental changes in mood state regulate immune function in relation to central opioid neurotransmission: a model of human CNS-peripheral inflammatory interaction. Molecular psychiatry 21(2): 243-251.

Raichle ME, MacLeod AM, Snyder AZ, Powers WJ, Gusnard DA, Shulman GL (2001). A default mode of brain function. Proceedings of the National Academy of 
Sciences of the United States of America 98(2): 676-682.

Rehbein F, Kliem S, Baier D, Mossle T, Petry NM (2015). Prevalence of Internet gaming disorder in German adolescents: diagnostic contribution of the nine DSM-5 criteria in a state-wide representative sample. Addiction 110(5): 842-851.

Riedl V, Utz L, Castrillon G, Grimmer T, Rauschecker JP, Ploner M, et al (2016). Metabolic connectivity mapping reveals effective connectivity in the resting human brain. Proceedings of the National Academy of Sciences of the United States of America 113(2): 428-433.

Sheline, Y. I., Price, J. L., Yan, Z., \& Mintun, M. A. (2010a). Resting-state functional mri in depression unmasks increased connectivity between networks via the dorsal nexus. Proceedings of the National Academy of Sciences, 107(24), 11020.

Sheline YI, Raichle ME, Snyder AZ, Morris JC, Head D, Wang S, et al (2010b). Amyloid plaques disrupt resting state default mode network connectivity in cognitively normal elderly. Biological psychiatry 67(6): 584-587.

Shi H, Wang X, Yi J, Zhu X, Zhang X, Yang J, et al (2015). Default mode network alterations during implicit emotional faces processing in first-episode, treatment-naive major depression patients. Frontiers in psychology 6: 1198.

Shirer WR, Ryali S, Rykhlevskaia E, Menon V, Greicius MD (2012). Decoding subject-driven cognitive states with whole-brain connectivity patterns. Cerebral cortex 22(1): 158-165.

Tao, H., Guo, S., Ge, T., Kendrick, K. M., Xue, Z., Liu, Z., \& Feng, J. (2013). Depression uncouples brain hate circuit. Molecular psychiatry, 18(1), 101-111.

Thompson GJ, Riedl V, Grimmer T, Drzezga A, Herman P, Hyder F (2016). The whole-brain "global" signal from resting state fMRI as a potential biomarker of quantitative state changes in glucose metabolism. Brain connectivity. 
Veer, I. M., Beckmann, C., Van Tol, M. J., Ferrarini, L., Milles, J., Veltman, D.,Aleman, A., Van Buchem M. A., Van der Wee, N. J., \& Rombouts, S. A. (2010). Whole brain resting-state analysis reveals decreased functional connectivity in major depression. Frontiers in systems neuroscience, 4, (41),1-10.

Wager TD, Davidson ML, Hughes BL, Lindquist MA, Ochsner KN (2008). Prefrontal-subcortical pathways mediating successful emotion regulation. Neuron 59(6): 1037-1050.

Wang, L., Wu, L., Lin, X., Zhang, Y., Zhou, H., Du, X., \& Dong, G. (2016). Altered brain functional networks in people with Internet gaming disorder: Evidence from resting-state fMRI. Psychiatry Research: Neuroimaging, 254, 156-163.

Wee, C. Y., Zhao, Z., Yap, P. T., Wu, G., Shi, F., Price, T., Du, Y., Xu, J., Zhou, Y., \& Shen, D. (2014). Disrupted brain functional network in internet addiction disorder: a resting-state functional magnetic resonance imaging study. PloS one, 9(9), e107306.

Wei L, Duan X, Zheng C, Wang S, Gao Q Zhang Z, et al (2014). Specific frequency bands of amplitude low-frequency oscillation encodes personality. Human brain mapping 35(1): 331-339.

Yan C-G, Zang Y-F (2010). DPARSF: A MATLAB Toolbox for "Pipeline" Data Analysis of Resting-State fMRI. Frontiers in systems neuroscience 4: 13.

Yan, C. G., Cheung, B., Kelly, C., Colcombe, S., Craddock, R. C., Di Martino, A., ... \& Milham, M. P. (2013). A comprehensive assessment of regional variation in the impact of head micromovements on functional connectomics. Neuroimage, 76, 183-201.

Young KS (2009). Internet Addiction Test (IAT). 
Yuan K, Yu D, Cai C, Feng D, Li Y, Bi Y, et al (2016). Frontostriatal circuits, resting state functional connectivity and cognitive control in internet gaming disorder. Addiction biology.

Zang Y, Jiang T, Lu Y, He Y, Tian L (2004). Regional homogeneity approach to fMRI data analysis. Neuroimage 22(1): 394-400.

\section{Figure legends}

Figure 1. The ROIs comprising the ventral and dorsal DMN networks

(a) The ventral DMN includes four brain regions including the posterior cingulate cortex (PCC), precuneus, medial frontal gyrus (MFG) and medial temporal gyrus (MTG); (b) The dorsal DMN includes four brain regions within the PCC, MFG, medial cingulate cortex (MCC) and right thalamus. These templates were downloaded from Stanford's Functional Imaging in Neuropsychiatric Disorders lab (http://findlab.stanford.edu/functional ROIs.html), and permission to print the figure was received.

\section{Figure 2. Correlations between PoMS scores and FCs among ventral DMN regions}

Significant negative correlations were found between the PoMS Depression scores and FCs among ventral DMN brain regions. Marginally significant negative correlation was found between FC of the ventral DMN and the total PoMS score. 
One subject scored a 49 on the Tension measure and was viewed as an outlier. The PoMS data from this subject were involved in all correlation analyses except those relating to Tension.

Figure 3. Correlations between PoMS scores and FCs among dorsal DMN regions

Significant negative correlations were found between Anger scores and FCs among dorsal DMN brain regions. One subject scored a 49 on the Tension measure and was viewed as an outlier. The PoMS data from this subject were involved in all correlation analyses except those relating to Tension.

Figure 4. Correlations between PoMS scores and FCs among both ventral and dorsal DMN regions.

Negative correlations were found between FCs of the ventral and dorsal DMN and Depression and Anger scores. One subject scored a 49 on the Tension measure and was viewed as an outlier. The PoMS data from this subject were involved in all correlation analyses except those relating to Tension.

Figure 5. PoMS scores and FCs among DMN in differentiating IGD and HC.

(a) PoMS scores in IGD and HC groups; (b) FCs among DMN networks in IGD and HC groups. ${ }^{* *} p<0.01{ }^{*} p<0.05$. 


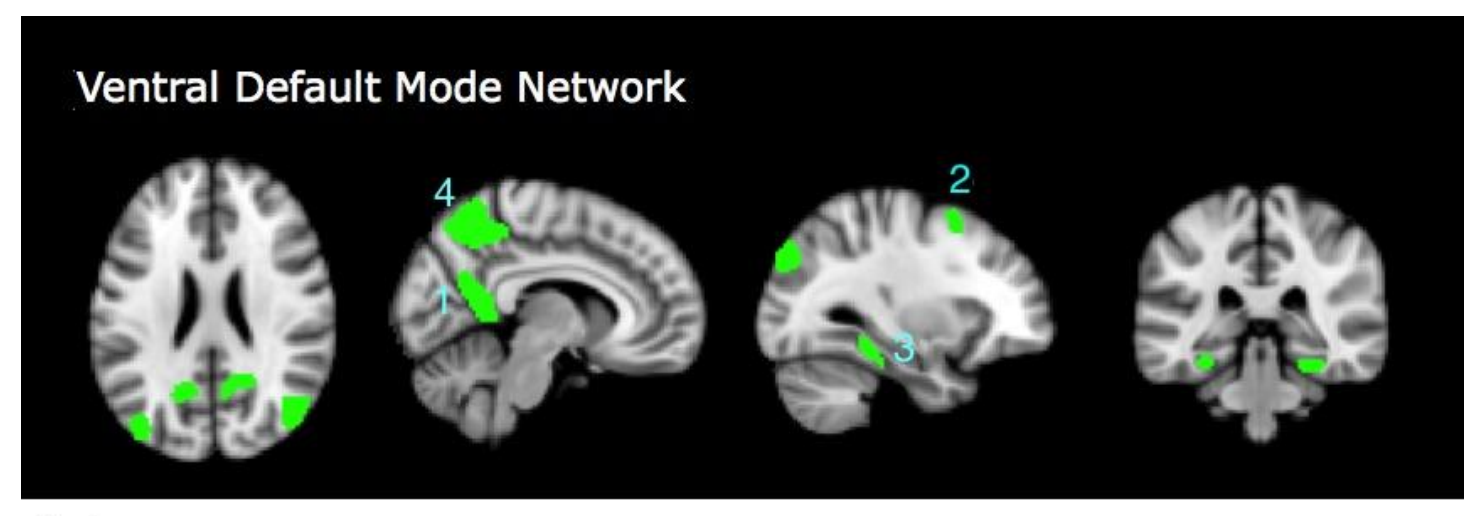

(a) $1 \mathrm{PCC} 2 \mathrm{MFG} 3 \mathrm{MTG} 4$ Precuneus

Dorsal Default Mode Network

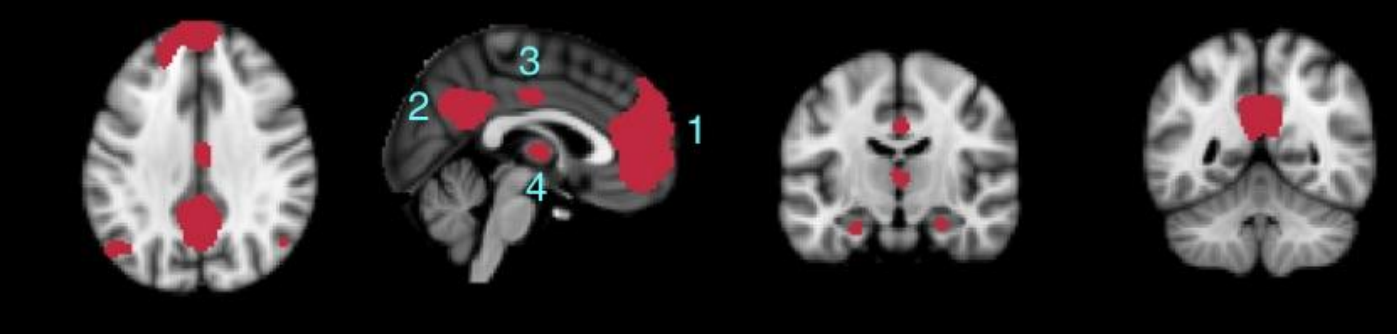

(b) 1 MFG 2 PCC 3 MCC 4 Thalamus 

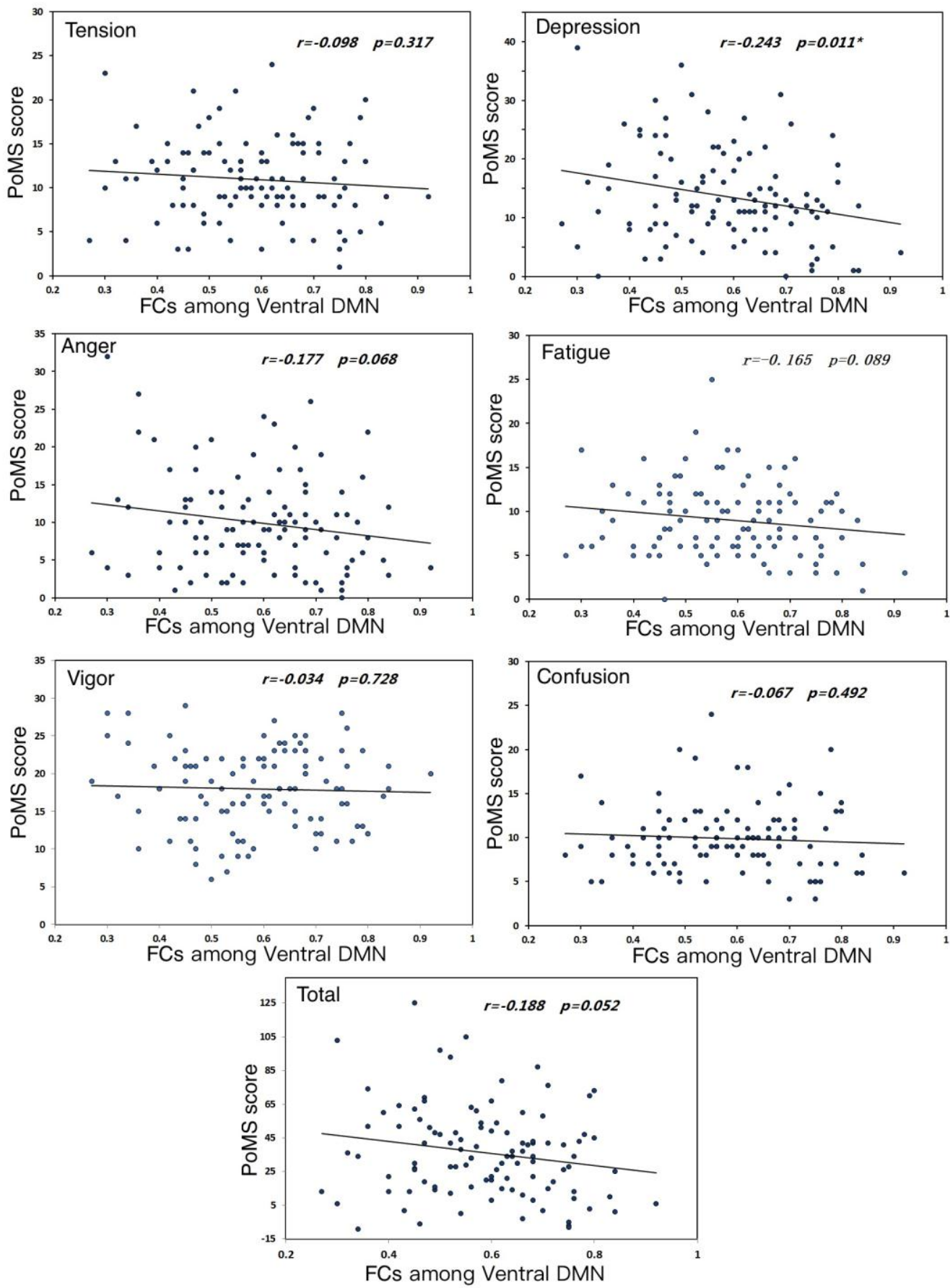

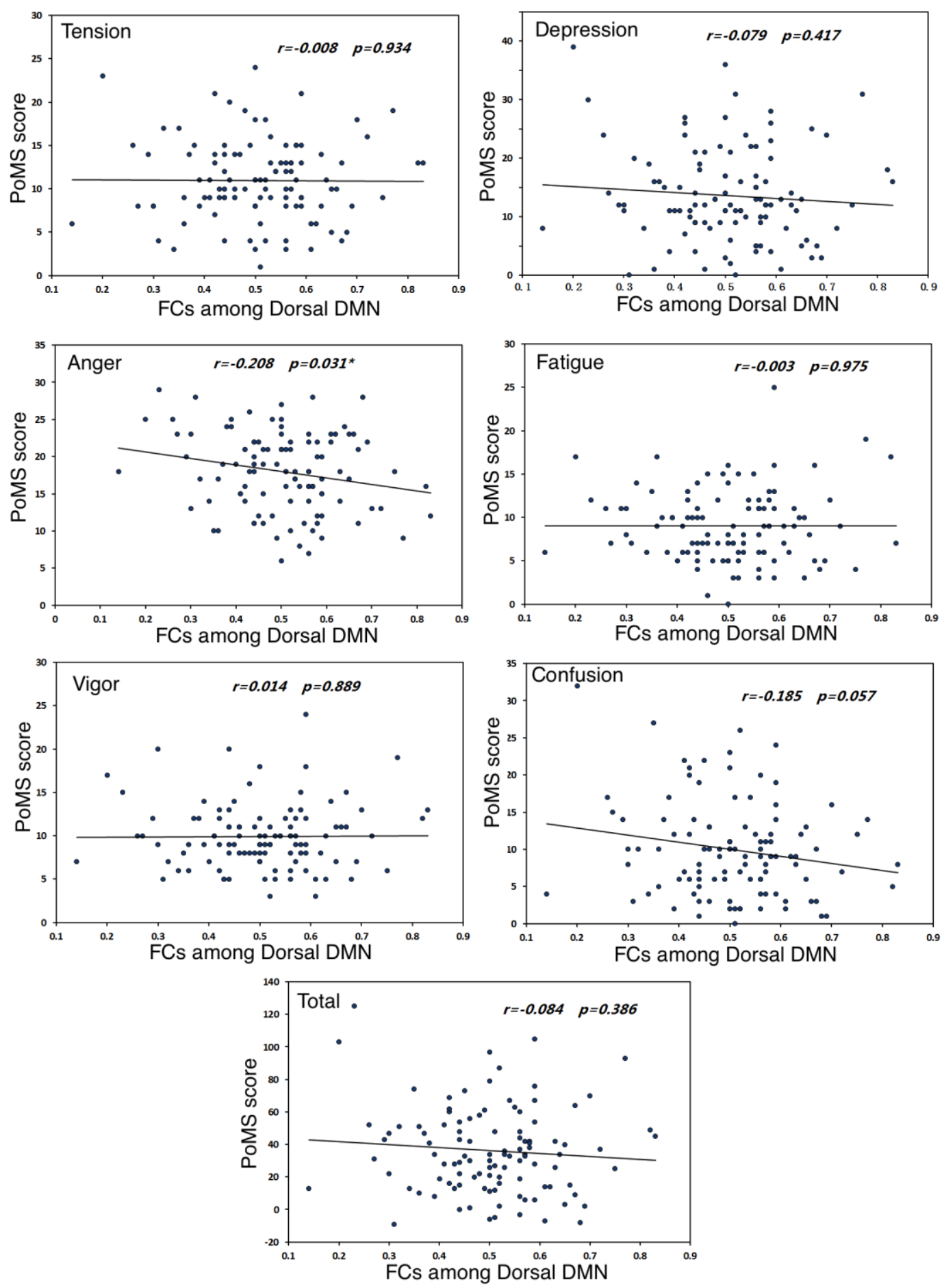

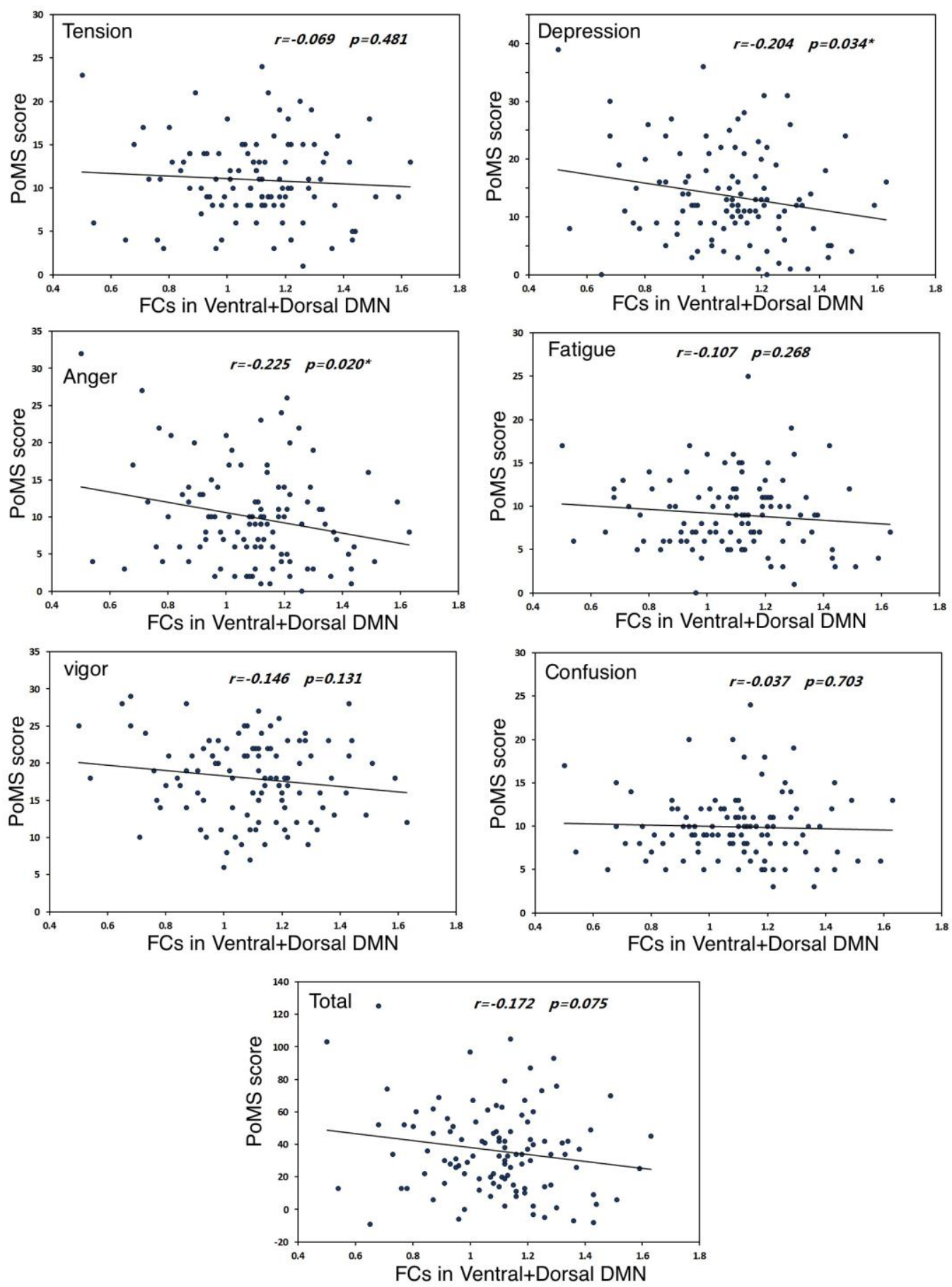

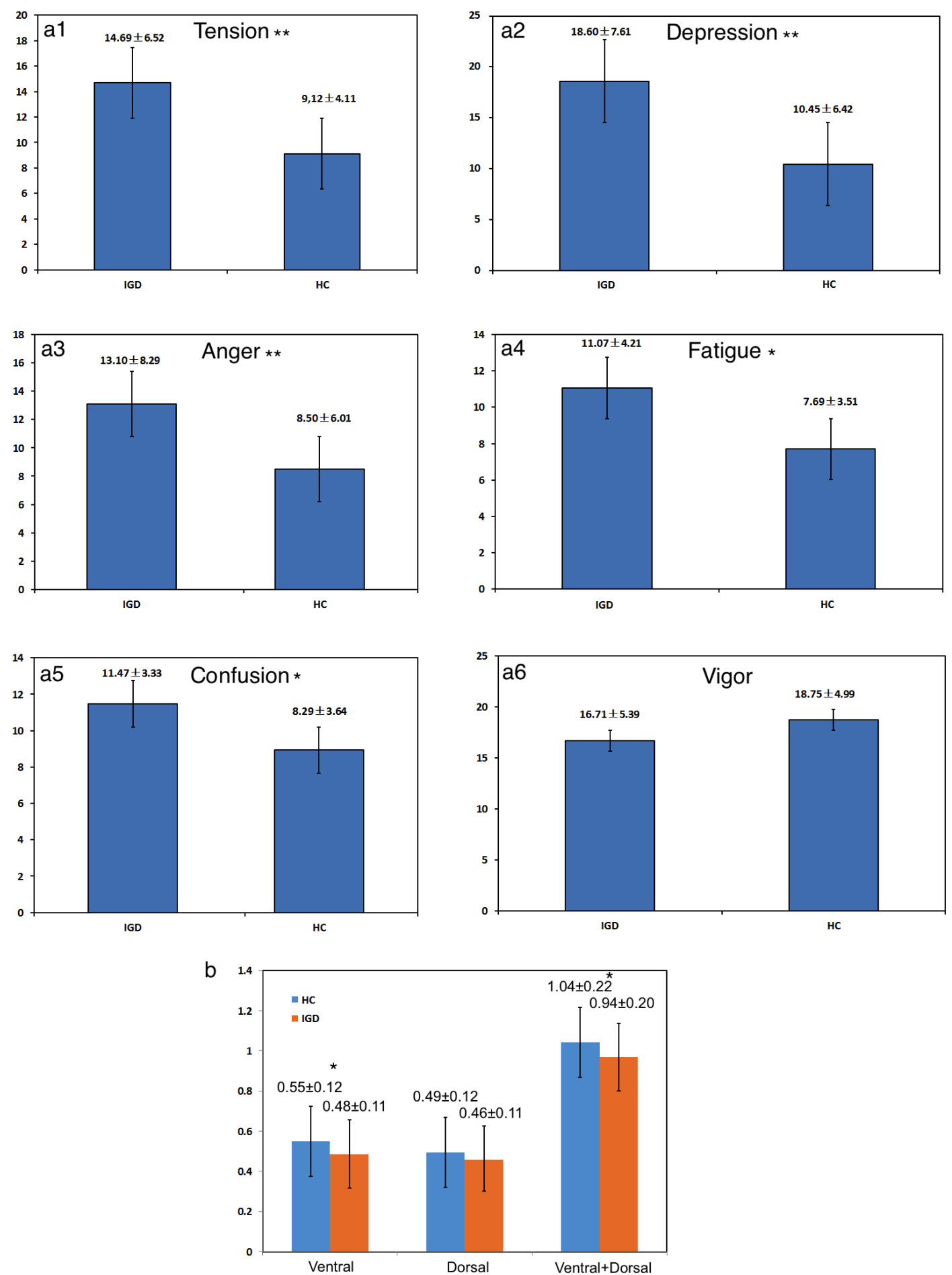